\title{
Dendritic cell-associated B7-H3 suppresses the production of autoantibodies and renal inflammation in a mouse model of systemic lupus erythematosus
}

\author{
Xu Zheng ${ }^{1,2}$, Ze Xiu Xiao², Li Hu², Xuan Fang ${ }^{1}$, Liqun Luo ${ }^{2,3}$ and Lieping Chen $\mathbb{1}^{2,3,4}$
}

\begin{abstract}
B7-H3 immune modulatory molecule has been implicated in the generation and pathogenesis of autoimmune diseases, the mechanism of action is less known. We explored the role of $\mathrm{B} 7-\mathrm{H} 3$ in the induction of autoantibodies and organ-directed inflammation in a murine systemic lupus erythematosus (SLE) model in which the immunization with DNA extracted from activated T cells induced the production of anti-DNA autoantibodies and subsequent glomerulonephritis, two hallmarks of human SLE. Mice deficient of B7-H3 or treated with a B7-H3 specific antibody produced significantly higher levels of anti-DNA autoantibodies and more severe glomerulonephritis than wild-type mice, indicating an inhibitory function of B7-H3 in this model. Interestingly, immunization of mice with DNA-pulsed dendritic cells induced severe SLE symptoms while B7-H3 on dendritic cells is required in this process. Importantly, treatment of mice with recombinant B7-H3lg fusion protein effectively ameliorated progression of murine SLE, accompanied with decreased level of anti-DNA autoantibodies and alleviated glomerulonephritis, decreased autoantibody deposition and complement deposition in kidney. Our findings implicate a potential role of B7-H3 on dendritic cells in the induction of SLE and as a potential target for the treatment of autoimmune diseases.
\end{abstract}

\section{Introduction}

B7-H3, a cell surface molecule of the extended CD28/B7 family, was discovered in our laboratory in 2001 by searching databases for molecules with homology to previously identified $\mathrm{B} 7$ molecules $^{1}$. B7-H3 has single IgVand IgC-like domains (2Ig form) with a transmembrane and intracellular tail in human, mice and other species ${ }^{2}$. In human, a unique isoform with dual IgV- and IgC-like

\footnotetext{
Correspondence: Xu Zheng (827999120@qq.com) or Lieping Chen (lieping. chen@yale.edu)

'Department of Rheumatology \& Immunology, The First Affiliated Hospital of USTC, Division of Life Sciences and Medicine, University of Science and Technology of China, Hefei, AH, P. R. China

${ }^{2}$ Immunotherapy Laboratory, Sun Yat-Sen University, Guangzhou, GD, P. R. China

Full list of author information is available at the end of the article.

These authors contributed equally: Xu Zheng, Ze Xiu Xiao

Edited by $Y$. Shi
}

domains (4Ig form) was also identified ${ }^{3}$. B7-H3 mRNA is broadly detected in normal tissues and its cell surface expression, although much rare, could also be found on activated DCs in lymphoid tissue ${ }^{4,5}$. While immunological function of $\mathrm{B} 7-\mathrm{H} 3$ is yet to be elucidated, early studies indicate that $\mathrm{B} 7-\mathrm{H} 3$ co-stimulates human and murine $\mathrm{T}$ cell proliferation and cytokine secretion in vitro and enhances tumor rejection by over-expression in tumor lines in animal models ${ }^{1,6,7}$. Whereas these studies suggest a positive role of $\mathrm{B} 7-\mathrm{H} 3$ in the regulation of $\mathrm{T}$ cell responses, other studies, however, indicate that $\mathrm{B} 7-\mathrm{H} 3$ also have inhibitory functions on several $\mathrm{T}$ cell responses, including inhibition of $\mathrm{T}$ cell proliferation in vitro and allergen-induced autoimmunity in murine models ${ }^{8}$. Currently there are several possible interpretations for these seeming contradictory roles of $\mathrm{B} 7-\mathrm{H} 3$ in immune responses. A hypothesis for these seemingly contradictory

\section{(c) The Author(s) 2019}

(c) (i) Open Access This article is licensed under a Creative Commons Attribution 4.0 International License, which permits use, sharing, adaptation, distribution and reproduction in any medium or format, as long as you give appropriate credit to the original author(s) and the source, provide a link to the Creative Commons license, and indicate if changes were made. The images or other third party material in this article are included in the article's Creative Commons license, unless indicated otherwise in a credit line to the material. If material is not included in the article's Creative Commons license and your intended use is not permitted by statutory regulation or exceeds the permitted use, you will need to obtain permission directly from the copyright holder. To view a copy of this license, visit http://creativecommons.org/licenses/by/4.0/. 
data is that differential role of $\mathrm{B} 7-\mathrm{H} 3$ in the regulation of distinct $\mathrm{T}$ cell subsets ${ }^{9}$. Another possible explanation is the engagement of different putative receptors by B7-H3.

Systemic lupus erythematosus (SLE) is a systemic autoimmune disease and is characterized by the presence of hyperactive immune cells and aberrant antibody responses to nuclear and cytoplasmic antigens, including characteristic anti-double-stranded DNA antibodies (antidsDNA Abs $)^{10-12}$. Anti-dsDNA Abs are thought to be diagnostic markers in SLE and their presence in humans and mice often correlates with disease pathogenesis ${ }^{13-16}$. Furthermore, these Abs also contribute to disease progression of SLE, as indicated by glomerulonephritis, an inflammation largely due to deposition of antigen (Ag)Abs complex and subsequent activation of complement. Qiao et al. ${ }^{17}$ showed that mice immunized with activated lymphocyte-derived DNA (ALD-DNA) produced high levels of anti-dsDNA Abs, and subsequently developed SLE-like syndrome, including aggravated glomerulonephritis, increased autoantibody and complement deposition. These observations resemble closely human SLE and this may provide a good model for studying human SLE pathogenesis.

Dendritic cells (DCs) are showed to be functionally abnormal in human SLE, including a reduced number of circulating conventional DCs, but increased plasmatoid DC (pDCs) $)^{18}$. In SLE, conventional DCs promote autoreactivity rather than tolerance ${ }^{19}$. In turn, activated $\mathrm{T}$ cells also promote increased type I interferon (IFN) production by $\mathrm{pDCs}^{20}$. Conventional DCs were also shown to contribute to the development of lupus nephritis in a mouse model $^{21}$. Collectively, these findings implicate possible contribution of DCs to the disease progression of SLE. B7$\mathrm{H} 3$ is not expressed in significant amounts on freshly isolated lymphocytes but could be induced on DCs and monocytes/macrophages upon activation ${ }^{22}$. Expression of B7-H3 on DCs could be further enhanced by the TH1 cytokine IFN- $\gamma$ or $\mathrm{LPS}^{5}$. The function of B7-H3 on DCs, however, is unclear.

In this study, we show that mouse B7-H3 on DCs has a negative regulatory function for $\mathrm{CD} 4+\mathrm{T}$ cell-dependent production of anti-dsDNA Abs and contributes to disease progression in a murine SLE model.

\section{Materials and methods}

\section{Mice strains and cell lines}

Female C57BL/6 (B6) mice, littermate control mice and B7-H3 knockout (KO) mice ${ }^{9}$ were used in aged 6-10 weeks. B7-H3KO mice were generated in Chen's laboratory and have been backcrossed to B6 background for 10 generations. B6 lpr/lpr were purchased from Model Animal Research Center of Nanjing University. B6 lpr/lpr $\times$ B7$\mathrm{H} 3 \mathrm{KO}$ (B6 lpr/lpr-KO) mice were obtained by backcrossing between $\mathrm{B} 6 \mathrm{lpr} / \mathrm{lpr}$ and $\mathrm{B} 7-\mathrm{H} 3 \mathrm{KO}$ and all mice were housed in a specific pathogen-free (SPF) room. Mouse housing procedures were conducted according to the Guide for the Care and Use of Medical Laboratory Animals (Ministry of Health, PRC, 1998). The DC2.4 dendritic cell line (H-2 $)$ originated from a $\mathrm{B} 6$ mouse was developed by superinfecting GM-CSF-transduced bone marrow cells with myc and raf oncogenes ${ }^{23}$. For cell transfection, 293T cells were seeded at $8 \times 10^{6}$ on $10 \mathrm{~cm}$ dish and transfected at next day when they were $70-80 \%$ confluency. Prior to transfection, the culture medium was changed with DMEM. Mix with 30ug plasmid B7-H3Ig or control Flag-Ig and 90ug PEI incubating for $15 \mathrm{~min}$ in Opti-MEM. After adding the complexes to the dishes for 5-6 days, supernatant was collected for further purification.

\section{Detection of anti-DNA autoantibodies}

IgG antibodies to ALD-DNA were assessed using a previously described enzyme-linked immunosorbent assay (ELISA) with modification ${ }^{24}$. Briefly, 96-well plates were coated with double stranded salmon sperm DNA $100 \mu \mathrm{g} / \mathrm{well}$ at $100 \mu \mathrm{g} / \mathrm{ml}$ and placed overnight at $4{ }^{\circ} \mathrm{C}$. The plates were then washed four times with PBS containing $0.05 \%$ Tween-20, dried and were added $200 \mu \mathrm{l}$ of blocking solution (PBS containing 10\% FBS) per well. Mouse serum diluted 1:50 in PBS containing 1\% BSA was added to each well and incubated for $2 \mathrm{~h}$ at $37^{\circ} \mathrm{C}$. The plates were washed 10 times, followed by the addition of anti-mouseIgG1-Fc-HRP (horseradish peroxidase) specific secondary antibodies which were diluted 1:4000 in PBS containing 1\% FBS $100 \mu \mathrm{l} /$ well. After extensive washing, the plates were incubated for $20 \mathrm{~min}$ with $100 \mu \mathrm{l} /$ well TMB. The absorbance was measured at $450 \mathrm{~nm}$ on a microtiter plate reader (Southern Biotech, USA).

\section{Flow cytometry analysis and antibodies}

The monoclonal antibody $(\mathrm{mAb})$ clone $14 \mathrm{M}$ is a mouse anti-mouse IgG1 mAb against $\mathrm{B} 7-\mathrm{H} 3$ generated by the immunization of a $\mathrm{B} 7-\mathrm{H} 3 \mathrm{KO}$ mouse. $\mathrm{PE}$ anti-mouse CD8a, PE-cy7 or APCanti-mouse CD4, FITC anti-mouse $\mathrm{CD} 3$, PE anti-mouse CD11c and isotype-matched mAbs were purchased from BD Biosciences; and anti-p-STAT3 antibody was purchased from Cell Signaling Technology. Fixation/Permeabilization Kit were purchased from eBioscience. For cell surface staining, cells were directly stained with either IgG control or fluorescenceconjugated antibodies. For intracellular staining, cells were permeabilized and fixed after surface staining, and stained with fluorescence-conjugated antibodies or IgG controls.

\section{DNA immunization}

Genomic DNA was extracted from Con A-stimulated splenocytes (ALD-DNA) as described previously ${ }^{25}$. The concentration of ALD-DNA was determined by the 
absorbance (A) at $260 \mathrm{~nm}$, and the final A260/280 was $>1.8$. All procedures were practiced under aseptic conditions. Mice were subcutaneously (sc) injected under the dorsal skin with ALD-DNA $(50 \mu \mathrm{g} / \mathrm{mice})$ in complete Freund's adjuvant (sigma), followed by two booster immunizations $(50 \mu \mathrm{g} / \mathrm{mice})$ emulsified with incomplete Freund's adjuvant at weeks 2 and $4^{26}$. Mice were intraperitoneally (i.p.) injected with $14 \mathrm{M}$ or control Ig. Mice were bled from retro-orbital sinus before immunization and every 2 weeks after their initial immunization and sera were prepared for the assay. All mice were sacrificed 12 weeks post-immunization, spleen, lymph nodes and kidneys were collected for further analysis.

\section{Immunization by bone marrow-derived dendritic cell (BMDC)}

DCs were prepared from mouse bone marrow. Briefly, bone marrow cells were flushed from femur and tibia. The harvested cells were cultured in RPMI-1640 complete medium with $10 \%$ fetal bovine serum (Gibco) containing $50 \mathrm{ng} / \mathrm{ml}$ recombinant mouse ( $\mathrm{rm}$ ) GM-CSF (peProTech), $12.5 \mathrm{ng} / \mathrm{ml} \mathrm{rmIL-4(peProTech).} \mathrm{At} \mathrm{day} \mathrm{3,} \mathrm{fresh} \mathrm{medium}$ containing rmGM-CSF and rmIL-4 was added to each culture flask. At day 6, half of the cell suspension was centrifuged, suspended in the same volume of cell culture medium and returned to the culture flask. The cells were harvested and used for experiments. For immunization, BMDC were incubated with $5 \mathrm{ug} / \mathrm{ml}$ ALD-DNA overnight. After extensive wash, $5 \times 10^{5}$ BMDC-ALD-DNA were intravenously (i.v.) injected, followed by i.p. injection with $14 \mathrm{M}$ or control Ig. Mice were bled from retro-orbital sinus every 2 weeks and sacrificed at week 10 and spleen, lymph nodes and kidneys were collected for further analysis. For early stage treatment, $5 \times 10^{5}$ BMDC-ALD-DNA were i.v. injected, followed by i.p. injection with B7-H3Ig or control Ig at week 0. For late stage treatment, mice were treated with B7-H3Ig or control Ig at week 4 after the immunization with BMDC-ALD-DNA.

\section{Pathological assessment and evaluation of renal histopathology}

Kidneys from ALD-DNA-immunized mice were frozen in dry ice, embedded with OCT medium and $5 \mu \mathrm{m}$ sections were obtained and stained with hematoxylin (HE). Kidney sections assessed by a single observer, grading the kidneys for glomerular inflammation, proliferation, crescent formation, and necrosis. Interstitial changes and vasculitis were also noted. Scores from 0 to 3 were assigned for each of these features and then added together to yield a final renal scores. Glomerular inflammation was graded: 0 , normal; 1 , few inflammatory cells; 2, moderate inflammation; and 3, severe inflammation. Detailed pathological assessment was performed as described previously ${ }^{27}$.
Sections were stained with HRP-conjugated goat antimouse IgG (Sigma) or HRP-conjugated rabbit anti-mouse C3 (Sigma). Tissues from mouse kidneys were prepared and stained with HE and eosin using standard procedures.

\section{Cytometric bead array (CBA)}

The $14 \mathrm{M}$ or control Ig at $5 \mu \mathrm{g} / \mathrm{ml}$ was aspirated into the 12 -well plates overnight. After washing, $5 \times 10^{5}$ DC2.4 cells were added into the 12-well plates. Supernatant was collected $24 \mathrm{~h}$ later, and tested with mouse inflammation kit (BD). For the inflammation cytokine CBA assay, $50 \mu \mathrm{L}$ of supernatant were stained with the mixture of mouse cytokine capture bead suspension and the PE detection reagent. After $2 \mathrm{~h}$ of incubation, samples were washed and then analyzed by using the BD CBA software. Mouse inflammation cytokine standards provided with the kit were diluted and used in parallel to samples for preparation of the standard curves as instructed.

\section{Quantitative real-time PCR (qRT-PCR) assay}

Total RNA was extracted using RNeasy (Qiagen) and was reverse transcribed to cDNA using Taqman reverse transcription reagents (Applied Biosystems) according to the manufacturer's instructions. Primer and probe sets were obtained from Applied Biosystems. qRT-PCR was performed using the Taqman Universal PCR Master Mix. Primers used to amplify specific gene fragments as follow: B7-H3: 5'GACACGGATGCCACCCTACGCTG (forward) and 5'CTGTGATGGTGACTGAGCCGTGAG (reverse) and HPRT: 5'TCAACGGGGGACATAAAAGT (forward) and 5'TGCATTGTTTTACCAGTGTCAA (reverse). Expressions of B7-H3 were calculated by their ratios to HPRT.

\section{Statistical analysis}

The data are expressed as mean \pm standard deviation (SD). Two-tailed student's tests were used to calculate statistical significance. Data were shown as a representative experiment of three independent experiments. Statistical significance was defined as ${ }^{*} P<0.05,{ }^{* *} P<0.01$, and ${ }^{* * *} P<0.001$.

\section{Results}

Intrinsic B7-H3 suppresses the production of anti-DNA Abs and renal inflammation in a SLE mouse model

To evaluate possible role of $\mathrm{B} 7-\mathrm{H} 3$ in the production of autoantibodies and the pathogenesis of SLE, we first immunized B7-H3KO and WT B6 mice with activated lymphocyte-derived DNA (ALD-DNA). After the immunization, sera from mice were measured for the levels of anti-double stranded DNA antibodies (antidsDNA Abs) by specific ELISA. B7-H3KO mice had significantly higher levels of anti-dsDNA Abs than control wild-type mice in day 8, 10, and 12 (Fig. 1a), 
implicating that $\mathrm{B} 7-\mathrm{H} 3$ is inhibitory for the production of anti-dsDNA Abs in this model. We also tested the effect of a B7-H3 mAb (clone 14 M) in this model. Upon the immunization with ALD-DNA, WT B6 mice were treated with $14 \mathrm{M}$ or control immunoglobulin (Ig) and
4 weeks later, the levels of anti-dsDNA Abs in sera were tested by specific ELISA. Similar to B7-H3KO mice, the level of anti-dsDNA Abs in 14M-treated wild-type mice was significantly increased compared with those treated with control Ig (Fig. 1b). Flow cytometry analysis a

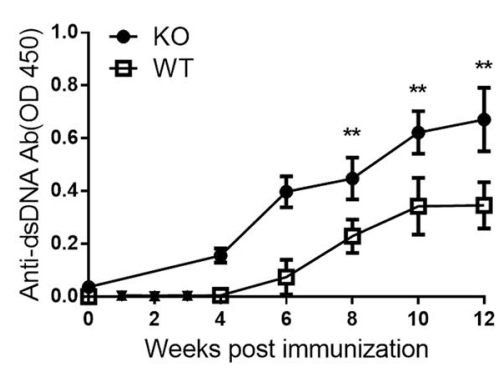

C

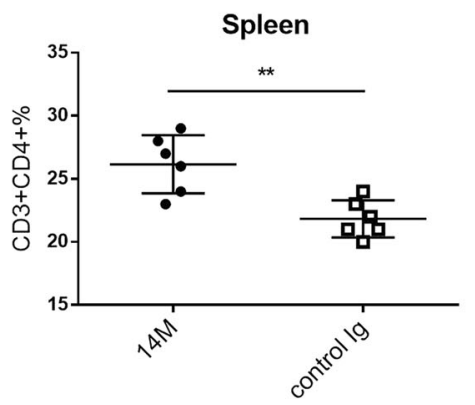

d

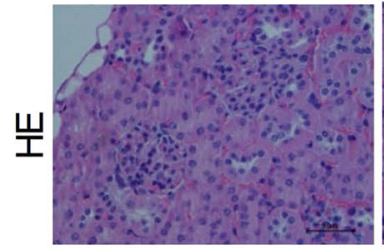

$14 \mathrm{M}$

e

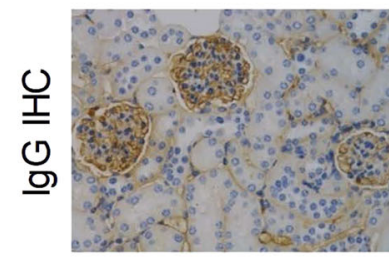

$14 \mathrm{M}$

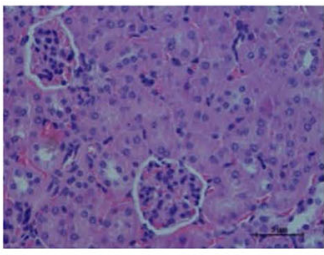

Control lg

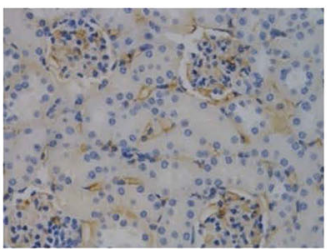

Control lg

b
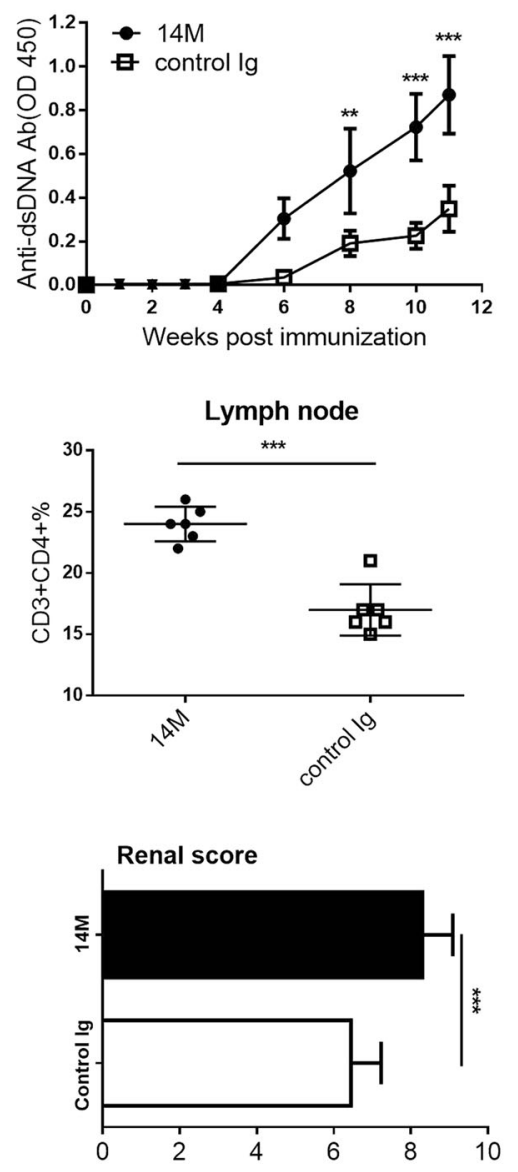

$f$

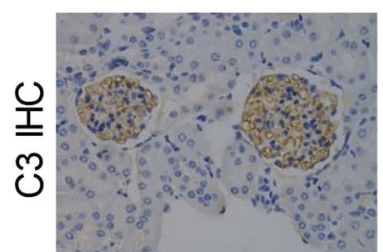

$14 \mathrm{M}$

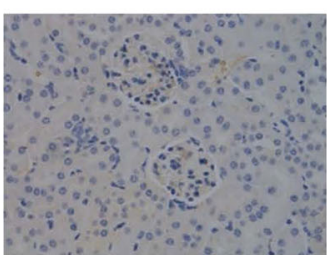

Control lg

Fig. 1 Intrinsic B7-H3 suppresses the production of anti-DNA Abs and renal inflammation in a SLE mouse model. a WT or B7-H3KO mice in groups of 5 were immunized with ALD-DNA (50ug/mouse) at 0, 2, 4 weeks. Sera from each mouse were collected at the indicated time points and anti-dsDNA Abs were analyzed by specific ELISA. $\mathbf{b}$ B6 mice in groups of 6 were immunized with ALD-DNA ( $50 \mu \mathrm{g} / \mathrm{mouse}$ ) at $0,2,4$ weeks and were treated i.p. with $14 \mathrm{M}$ or control Ig weekly. Anti-dsDNA Abs in sera were analyzed at the indicated time by ELISA. c. Four weeks after three ALD-DNA immunizations, CD3+CD4+ T cells in spleen (left panel) and lymph nodes (right panel) were numerated by flow cytometry with specific mAb in mice treated with $14 \mathrm{M}$ (filled circles) or control lg (hollow squares). Each circle or square represents the result for each mouse. $\mathbf{d}$-f Eight weeks after three ALD-DNA immunizations, mouse kidney sections were stained by hematoxylin and eosin (400X) (d left), the renal score of two groups (d right), IgG immunohistochemically staining (400x) (e), complement 3 immunohistochemically staining (400x) (f). Results are representatives of five mice 
showed that $14 \mathrm{M}$-treated mice had significantly higher levels of CD3 $+\mathrm{CD} 4+\mathrm{T}$ cells in both spleen (left panel) and lymph node (right panel) than those treated with control Ig (Fig. 1c). At week 12 after the immunization, we also evaluated kidney damage by tissue H\&E staining, autoantibody deposition and complement 3 deposition assays. Remarkably aggravated glomerulonephritis and increased autoantibody/complement depositions were observed in 14M-treated mice than the control (Fig. 1d-f). We also verified the function of B7H3 in B6 lpr/lpr-KO or B6 lpr/lpr -WT mice (Supplementary fig. 1a-d). The effect of $14 \mathrm{M}$ in B6 lpr/lpr model were also tested (Supplementary fig. 1e-g). Therefore, ablation of $\mathrm{B} 7-\mathrm{H} 3$ by either genetic $\mathrm{KO}$ or antibody blockade enhanced autoantibody production accompanied with more severe kidney impairment. Our data support that intrinsic B7-H3 suppresses autoantibody production and disease progression in this SLE model.

\section{Immunization with DNA-pulsed DCs induced SLE syndrome}

To test the role of DCs in the induction of SLE syndrome in our model, we first generated DC from bone marrow cells by in vitro cytokine stimulation (Fig. 2a) and subsequently pulsed the BMDC with ALD-DNA for $12 \mathrm{~h}$. Immunization of mice with BMDC-ALD-DNA led to the production of anti-dsDNA Abs in sera while immunization with BMDC without ALD-DNA did not induce anti-dsDNA Abs (Fig. 2b). Moreover, immunization with BMDC-ALD-DNA induced glomerulonephritis, autoantibody deposition and complement deposition whereas immunization with BMDC without DNA show normal kidney without significant autoantibody or complement deposition (Fig. 2c, e). Immunization with either ALD-DNA in adjuvants or BMDCALD-DNA induced high levels of anti-dsDNA Abs (Fig. 2f), indicating that ALD-DNA may be presented naturally by $\mathrm{DC}$ to stimulate anti-dsDNA Abs upon the immunization.

The production of anti-DNA Abs is CD4 $+\mathrm{T}$ cell-dependent

We next examined whether or not the production of anti-dsDNA Abs is dependent on CD4+ helper T cells. To test this, anti-CD4 mAb GK1.5 was injected into mice repeatedly to deplete $\mathrm{CD} 4+\mathrm{T}$ in vivo after the immunization with ALD-DNA. Using this method, we demonstrated that the depletion of $\mathrm{CD} 4+\mathrm{T}$ cells completely eliminated the production of anti-dsDNA Abs (Fig. 3a). Moreover, the depletion of CD4 $+\mathrm{T}$ cells also eliminated anti-dsDNA Abs production in the ALD-DNA-BMDC immunization model (Fig. 3b). Collectively, our results indicate that the production of anti-dsDNA Abs requires the help from $\mathrm{CD} 4+\mathrm{T}$ cells.

\section{DC-associated B7-H3 suppresses the production of anti- DNA Abs and renal inflammation}

To evaluate the role of $\mathrm{B} 7-\mathrm{H} 3$ in DC-mediated induction of anti-DNA autoantibodies, we first examined the expression of B7-H3 by BMDC. As shown, while resting BMDC expressed very low levels of $\mathrm{B} 7-\mathrm{H} 3$ in both protein or mRNA, incubation of BMDC with LPS significantly upregulated its expression (Fig. 4a), indicating that B7-H3 is largely an inducible molecule on DC.

To test the role of DC-associated $\mathrm{B} 7-\mathrm{H} 3$ in the induction of anti-DNA autoantibodies, mice were immunized with DC from either WT or B7-H3KO mice. The KO-BMDC-ALD-DNA induced significantly higher levels of anti-dsDNA Abs than WT-BMDC-ALD-DNA (Fig. 4b). Consistent to this finding, the treatment by $14 \mathrm{M}$ upon the immunization with WT-BMDC-ALDDNA increased anti-DNA Abs production (Fig. 4c) as well as aggravated glomerulonephritis, increased autoantibody deposition and complement deposition (Fig. $4 \mathrm{~d}-\mathrm{f}$ ). Our results indicate that DC-associated B7-H3 plays important role in the induction of anti-DNA Abs and inflammation.

\section{Blockade of B7-H3 enhances DC activation}

To determine the potential function of $\mathrm{B} 7-\mathrm{H} 3$ on DCs, an established cell line of DC (DC2.4) $)^{23}$ was used, which constitutively expressed high levels of $\mathrm{B} 7-\mathrm{H} 3$ as determined by flow cytometry after stained with anti-B7-H3 $\mathrm{mAb}$ (Fig. 5a). Interestingly, inclusion of $14 \mathrm{M}$ in the culture significantly promoted the production of IL-6 and TNF compared with the control Ig-treated DCs (Fig. 5b). It is well known that IL-6 expression is closely linked with STAT3 activation ${ }^{28}$ and there is a positive feedback loop between IL- 6 and STAT3 activation. We examined the levels of phosphorylation of STAT-3 (p-STAT-3) and showed that $14 \mathrm{M}$ treatment significantly increased the level of p-STAT-3 in DC2.4 cells in a dose dependent fashion compared with control Ig (Fig. 5c). These results suggest that intrinsic B7-H3 on DC2.4 may suppress IL-6 and TNF secretion and STAT-3 phosphorylation. Similar results were also obtained using BMDC: inclusion of $14 \mathrm{M}$ significantly enhanced the production of TNF and IL-6 from BMDC (Fig. 5d). Our findings thus indicate that blockade of $\mathrm{B} 7-\mathrm{H} 3$ promotes activation of DCs. Because DC2.4 is an established cell line and our findings implicate that $14 \mathrm{M}$ blocks a putative receptor on $\mathrm{DC}$ to interact with B7-H3.

\section{B7-H3 agonist ameliorates inflammatory symptoms in the SLE mouse model}

Our data above suggest that endogenous $\mathrm{B} 7-\mathrm{H} 3$ is suppressive for the initiation and development of lupuslike symptoms in the SLE mouse model. We next determined whether or not enforced expression of 


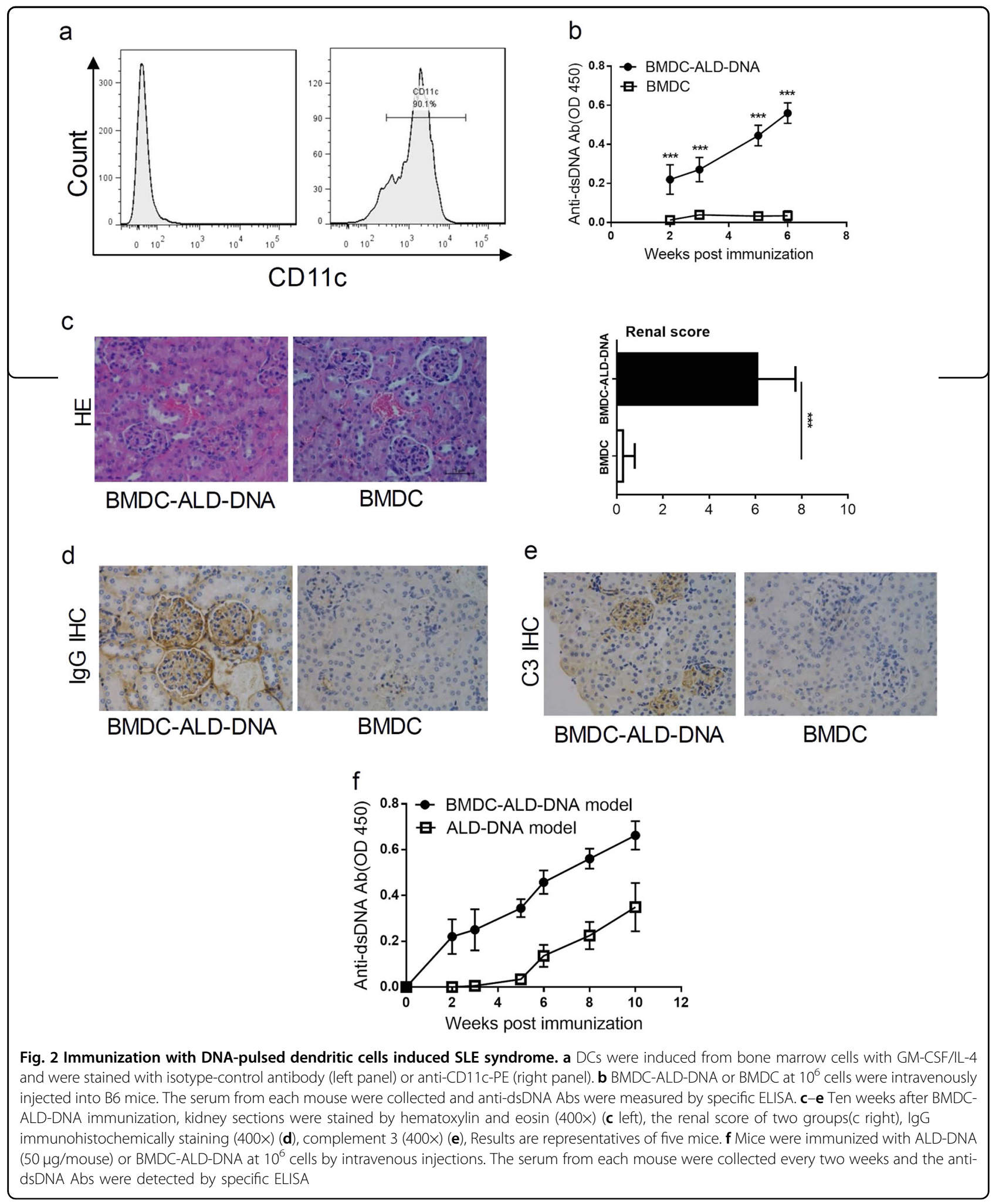

recombinant B7-H3Ig, as a agonist, could relieve murine SLE symptoms. To test this, mice were treated with B7H3Ig or control Ig after the immunization with BMDC-
ALD-DNA. The mice that received B7-H3Ig showed a significant decrease in anti-dsDNA Abs production compared with control Ig groups (Fig. 6a). Remarkably 

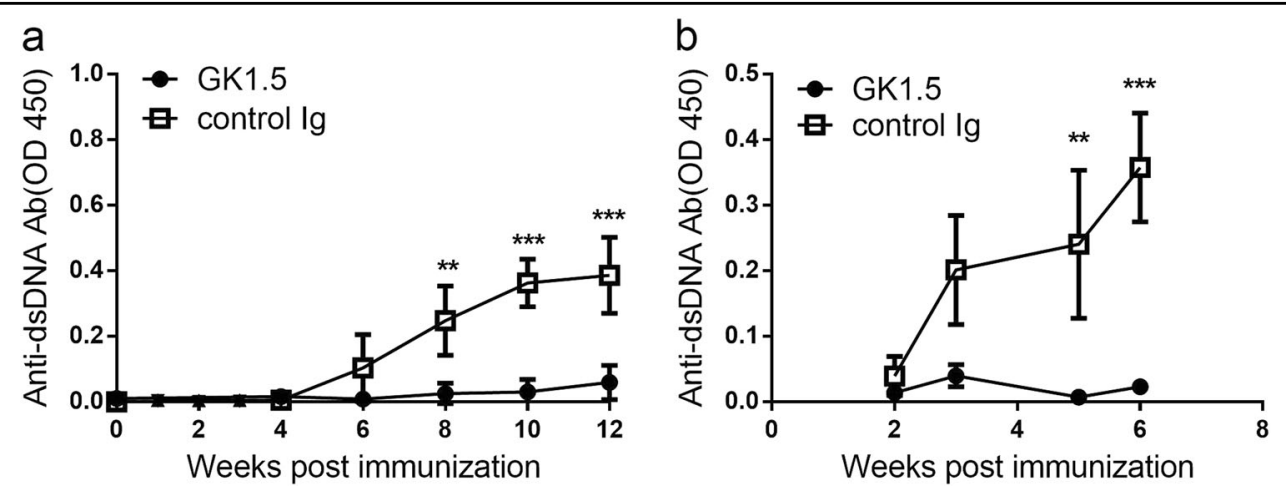

Fig. 3 The production of anti-DNA Abs is CD4+ T cell-dependent. Mice at groups of five were depleted with CD4+ T cells by the injection of GK1.5 mAb (700 $\mu \mathrm{g}$ per mouse) i.p. inoculated 2 days before ALD-DNA (a) or BMDC-ALD-DNA (10 cells) (b) immunization. The treatment of mice with GK1.5 were weekly. The serum from each mouse was collected and anti-dsDNA Abs were detected by specific ELISA

alleviated glomerulonephritis, decreased autoantibody deposition and complement deposition were found in B7H3Ig-treated SLE mice than control Ig treated SLE mice (Fig. 6b-d). However, mice were treated with B7-H3Ig after lupus injuries have occurred, the level of anti-dsDNA Abs in B7-H3Ig treatment was significant decrease compared with control Ig treatment (Supplementary fig. 2a) but renal score showed no difference (Supplementary fig. $2 \mathrm{~b})$ and the same degree of autoantibody deposition and complement deposition between two groups (Supplementary fig. 2c, d). Therefore, B7-H3Ig plays a more important in suppressing the progression of SLE disease at the early stage, especially in renal lesions. Our findings implicate a possible role of $\mathrm{B} 7-\mathrm{H} 3$ agonist as therapeutic agents for clinical SLE.

\section{Discussion}

By ablation of B7-H3, either genetic deficiency or blocking $\mathrm{mAb}$, our results indicate that $\mathrm{B} 7-\mathrm{H} 3$ may play an important role in the generation of anti-dsDNA Abs and subsequent promotion of renal inflammation in a SLE mouse model. Furthermore, we demonstrate that B7-H3 on DCs is required for the generation of anti-dsDNA Abs; while infusion of recombinant B7-H3Ig as agonist could suppress the production of anti-dsDNA Abs and ameliorate the disease progression in a mouse SLE model. Our findings implicate a role of B7-H3 pathway in the initiation and progression of human SLE.

DNA extracted from activated lymphocytes (ALDDNA) was shown to induce anti-dsDNA Abs and subsequent promotion of SLE-like symptoms, including chronic inflammation in kidney ${ }^{1729,30}$. DNA extracted from Con A-activated lymphocytes could trigger antidsDNA Abs production, leading to immune complex deposits in the kidney of syngeneic mice ${ }^{31}$. However, it is unclear how these DNA can immunize the host to generate anti-dsDNA Abs. In the present study we used BMDC which were coated with ALD-DNA, for the immunization and demonstrate their ability to trigger anti-dsDNA Ab in vivo. A widely used mouse strain for SLE model is MRL/lpr in which mice spontaneously develop autoantibodies and multiple organ impairments due to dysfunction of Fas/FasL cell death pathway ${ }^{32}$. B6 strain is not considered susceptible in general for the induction of autoantibodies and SLE. Our findings indicate that B6 mice could be induced to display SLE-like symptoms by the immunization with ALD-DNA and the ablation of B7-H3 promoted the progression of diseases. Currently clinical significance of these findings is yet to be explored. However, our findings suggest that decreased B7-H3 expression, especially on DCs, may be associated with more activated phenotypes of DCs and active SLE diseases and vice versa.

Constitutive expression of B7-H3 on a DC cell line, DC2.4, provides an opportunity to explore possible mechanisms of action. When $14 \mathrm{M}$ was included in the culture of DC2.4 cells, higher levels of IL-6 and TNF than control were detected and this activation is accompanied with increased expression of STAT3-P (Fig. 5). Similar findings were also obtained using freshly isolated BMDC culture in which $14 \mathrm{M}$ treatment could also promote IL-6 and TNF production (Fig. 5). Because in vivo experiments using the $\mathrm{KO}$ mice showed a role of $\mathrm{B} 7-\mathrm{H} 3$ in the suppression of autoantibody production, a major role of $14 \mathrm{M}$ and B7-H3Ig fusion protein should be interpreted as blocking effect. At the present time, however, we could not exclude the possibility that $14 \mathrm{M}$ may also have agonistic effect. It is possible that $14 \mathrm{M}$ has both antagonist and agonist effect on B7-H3 while the antagonist effect is dominant in vivo. In this context, these in vitro findings suggest the presence of a $\mathrm{B} 7-\mathrm{H} 3$ inhibitory receptor on DCs. While molecular nature of $\mathrm{B} 7-\mathrm{H} 3$ receptor on DCs 


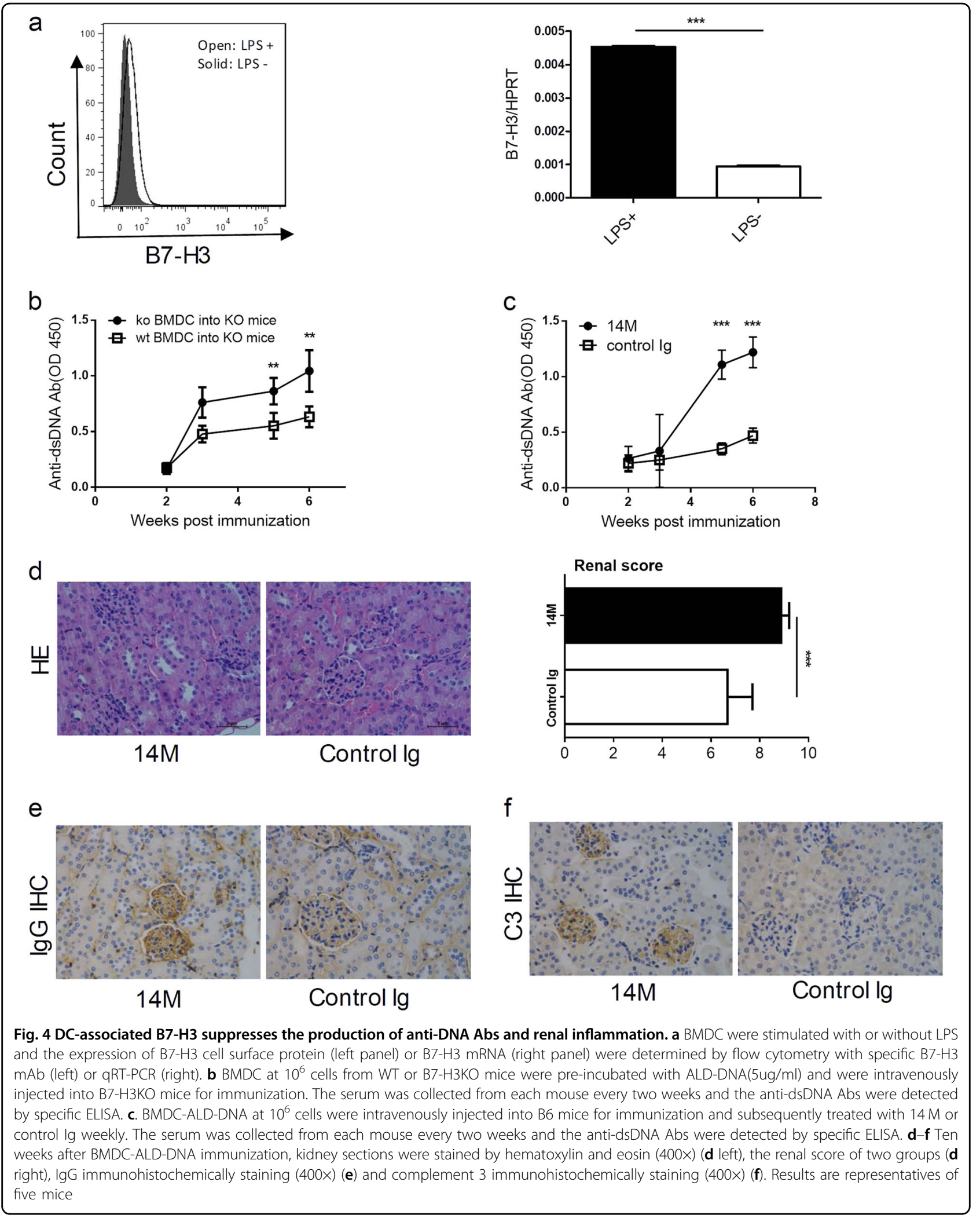




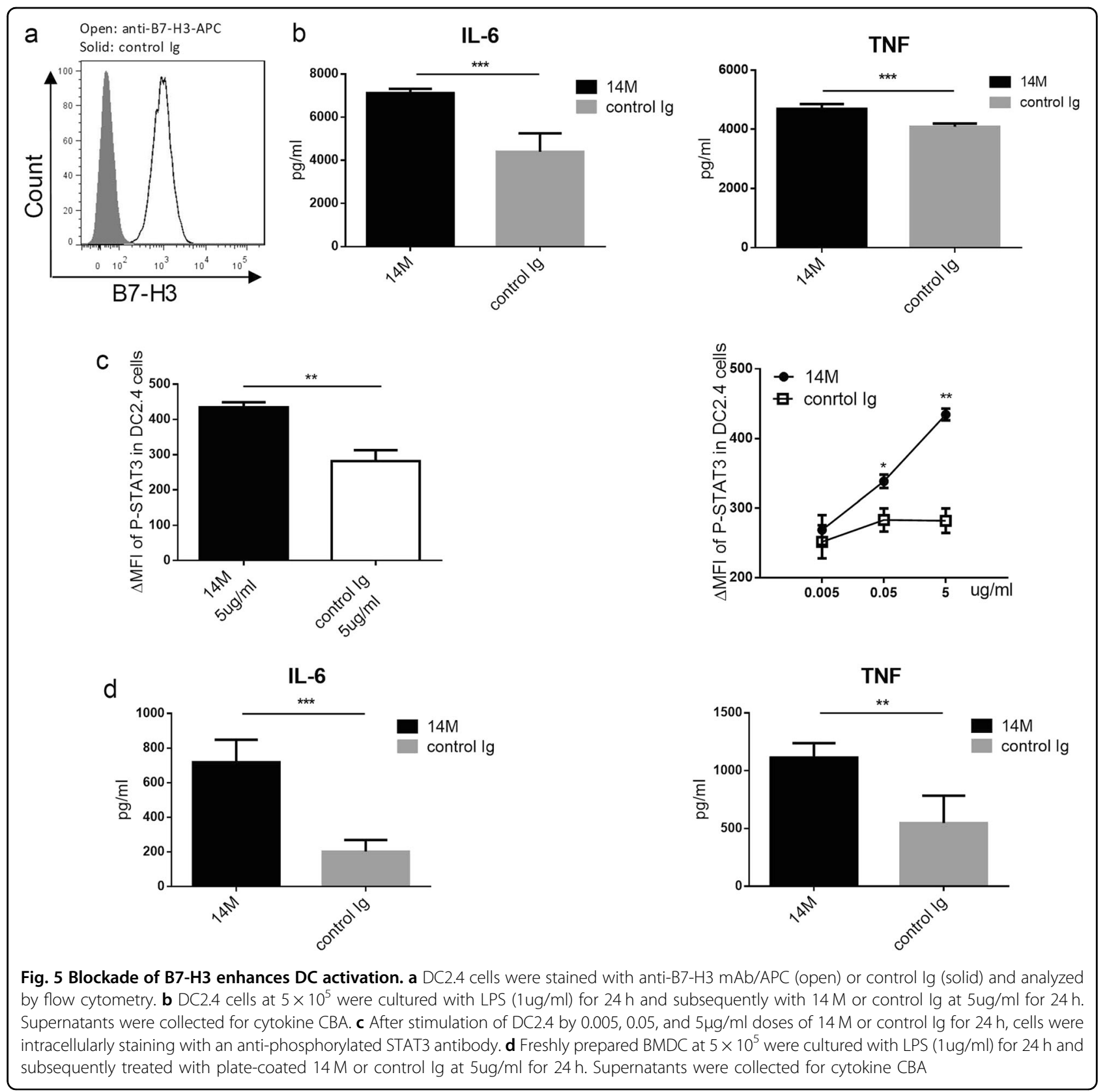

is yet to be identified, our findings have clinical implication since increased levels of IL-6 have been observed in SLE patients ${ }^{33}$ and its role in pathogenesis of SLE has been proposed ${ }^{34,35}$. Our findings are different from previously published studies suggesting the presence of B7$\mathrm{H} 3$ receptor on $\mathrm{T}$ cells ${ }^{36}$.

Administer of recombinant B7-H3Ig could attenuate clinical symptoms of SLE in our mouse model (Fig. 6). However, B7-H3Ig treatment after lupus injuries have occurred, can only alleviate the production of anti-dsDNA
Abs, but cannot reverse renal injury (Supplementary fig. 2 ). This finding is encouraging and indicates that $\mathrm{B} 7-\mathrm{H} 3$ could be potentially used for the treatment of SLE at the early stage. It is tempting to speculate that dimeric B7H3Ig engages its putative receptor on DCs and subsequently suppresses the induction of $\mathrm{CD} 4+\mathrm{T}$ cells so as to inhibit autoantibody production. Taken together, our study reveals a previously unknown suppressive mechanism of B7-H3 via DCs and proposes a possible approach for the treatment of human SLE. 


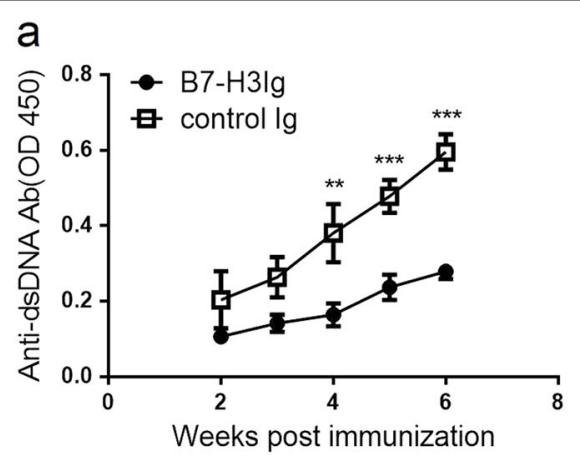

b

Ш

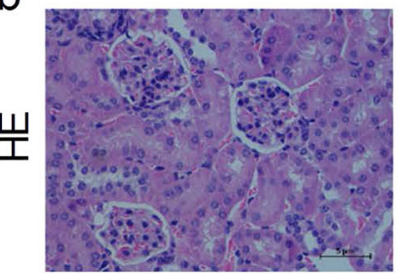

$\mathrm{B} 7-\mathrm{H} 3 \mathrm{lg}$

C

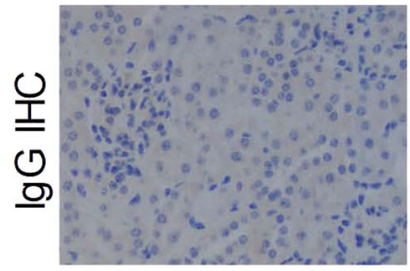

B7-H3lg

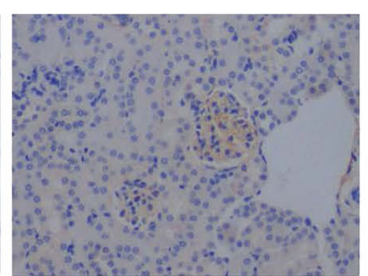

Control lg

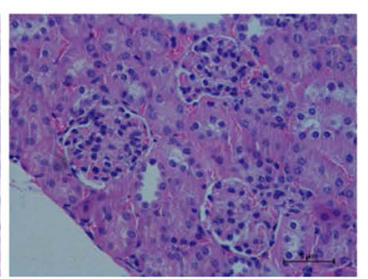

Control lg

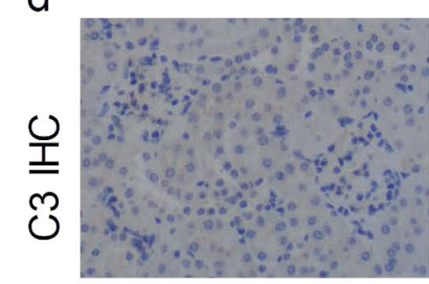

B7-H3lg

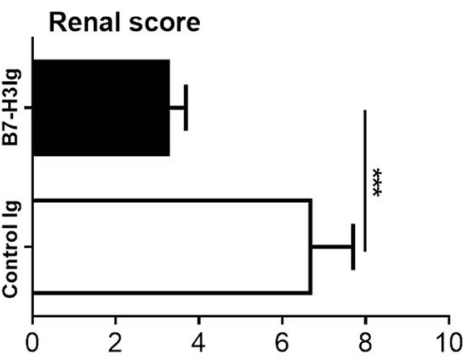

d

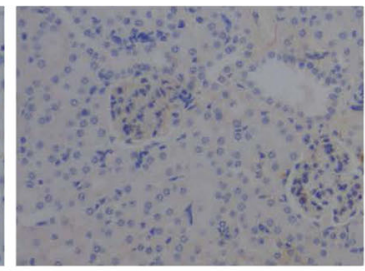

Control lg

Fig. 6 B7-H3 agonist ameliorates inflammatory symptoms in the SLE mouse model. a After the immunization with BMDC-ALD-DNA $\left(10^{6}\right)$, mice were treated with B7-H3 lg or control lg at $200 \mu \mathrm{g} / 0.5 \mathrm{ml}$ weekly and the anti-dsDNA Abs in sera were assayed by specific ELISA. b-d Ten weeks after $10^{6}$ BMDC-ALD-DNA immunization, kidney sections were stained by hematoxylin and eosin (400x) (b left), the renal score of two groups(b right), lgG immunohistochemically staining $(400 x)(\mathbf{c})$ and complement 3 immunohistochemically staining $(400 x)$ (d). Results are representatives of five mice

\section{Acknowledgements}

X.Z., Z.X., L.L., and L.C. developed the concept and designed the experiments. X.Z., Z.X., L.H., L.L., and L.C. performed and/or advised experiments. We thank Beth Cadugan for editing the manuscript. This study is partially supported by grants from the Fundamental Research Funds for the Central Universities (WK9110000052), Natural Science Foundation of Anhui Province

(1908085QH324), Guangdong Province Innovative Research Program Project (No. 2011Y035), 863 Project grants to Sun Yat-Sen University, Fujian Province Department of Science and Technology Research Program 2014Y2001 and 2014Y4008, the United Technologies Corporation endowed chair from Yale University and the Fundamental Research Funds for the Central Universities.

\section{Author details}

${ }^{1}$ Department of Rheumatology \& Immunology, The First Affiliated Hospital of USTC, Division of Life Sciences and Medicine, University of Science and Technology of China, Hefei, AH, P. R. China. ${ }^{2}$ Immunotherapy Laboratory, Sun Yat-Sen University, Guangzhou, GD, P. R. China. ${ }^{3}$ Immunotherapy Institute, Fujian Medical University, Fuzhou, FJ, P. R. China. ${ }^{4}$ Department of Immunobiology, Yale University, New Haven, CT, USA

\section{Statement of ethics}

Animal experiments conform to internationally accepted standards and have been approved by the appropriate institutional review body.

\section{Conflict of interest}

L.C. is an advisor/board member for Pfizer, NextCure, GenomiCare and Vcanbio and received research support from Boehringer Ingelheim, Pfizer and NextCure. LC is also an uncompensated adjunct faculty member of Sun YatSen University and Fujian Medical University. Other authors declare that they have no conflict of interest.

\section{Publisher's note}

Springer Nature remains neutral with regard to jurisdictional claims in published maps and institutional affiliations.

\section{Supplementary Information accompanies this paper at (https://doi.org/ 10.1038/s41419-019-1623-0).}

Received: 6 February 2019 Revised: 30 April 2019 Accepted: 3 May 2019 Published online: 21 May 2019

\section{References}

1. Chapoval, A. I. et al. B7-H3: a costimulatory molecule for T cell activation and IFN-gamma production. Nat. Immunol. 2, 269-274 (2001). 
2. Zhou, Y. H. et al. 4lgB7-H3 is the major isoform expressed on immunocytes as well as malignant cells. Tissue Antigens 70, 96-104 (2007).

3. Steinberger, P. et al. Molecular characterization of human 4lg-B7-H3, a member of the B7 family with four Ig-like domains. J. Immunol. 172 2352-2359 (2004).

4. Sun, M. et al. Characterization of mouse and human B7-H3 genes. J. Immunol. 168, 6294-6297 (2002).

5. Suh, W. K. et al. The B7 family member B7-H3 preferentially down-regulates T helper type 1-mediated immune responses. Nat. Immunol. 4, 899-906 (2003).

6. Luo, L. et al. B7-H3 enhances tumor immunity in vivo by costimulating rapid clonal expansion of antigen-specific CD8+ cytolytic T cells. J. Immunol. 173 5445-5450 (2004)

7. Sun, $\mathrm{X}$. et al. Mouse B7-H3 induces antitumor immunity. Gene Ther. 10 1728-1734 (2003).

8. Prasad, D. V. et al. Murine B7-H3 is a negative regulator of T cells. J. Immunol. 173, 2500-2506 (2004).

9. Luo, L. et al. B7-H3 promotes pathogenesis of autoimmune disease and inflammation by regulating the activity of different $T$ cell subsets. PLOS ONE 10, e0130126 (2015).

10. Swaak, A. J. et al. Prognostic value of anti-dsDNA in SLE. Ann. Rheum. Dis. 41, 388-395 (1982).

11. von Muhlen, C. A. \& Tan, E. M. Autoantibodies in the diagnosis of systemic rheumatic diseases. Semin. Arthritis Rheum. 24, 323-358 (1995).

12. Rahman, A. \& Isenberg, D. A. Systemic lupus erythematosus. N. Engl. J. Med. 358, 929-939 (2008).

13. Madaio, M. P. et al. Murine monoclonal anti-DNA antibodies bind directly to glomerular antigens and form immune deposits. J. Immunol. 138, 2883-2889 (1987).

14. Vlahakos, D. et al. Murine monoclonal anti-DNA antibodies penetrate cells, bind to nuclei, and induce glomerular proliferation and proteinuria in vivo. $J$. Am. Soc. Nephrol. 2, 1345-1354 (1992).

15. Suzuki, N. et al. Possible pathogenic role of cationic anti-DNA autoantibodies in the development of nephritis in patients with systemic lupus erythematosus. J. Immunol. 151, 1128-1136 (1993).

16. Lefkowith, J. B. \& Gilkeson, G. S. Nephritogenic autoantibodies in lupus: current concepts and continuing controversies. Arthritis Rheum. 39, 894-903 (1996).

17. Qiao, B. et al. Induction of systemic lupus erythematosus-like syndrome in syngeneic mice by immunization with activated lymphocyte-derived DNA. Rheumatology 44, 1108-1114 (2005).

18. Jin, O. et al. Systemic lupus erythematosus patients have increased number of circulating plasmacytoid dendritic cells, but decreased myeloid dendritic cells with deficient CD83 expression. Lupus 17, 654-662 (2008).

19. Mozaffarian, N., Wiedeman, A. E. \& Stevens, A. M. Active systemic lupus erythematosus is associated with failure of antigen-presenting cells to express programmed death ligand-1. Rheumatology 47, 1335-1341 (2008).
20. Leonard, D. et al. Activated T cells enhance interferon-alpha production by plasmacytoid dendritic cells stimulated with RNA-containing immune complexes. Ann. Rheum. Dis. 75, 1728-1734 (2016).

21. Celhar, T. et al. RNA sensing by conventional dendritic cells is central to the development of lupus nephritis. Proc. Natl Acad. Sci. USA 112, E6195-E6204 (2015).

22. Yi, K. H. \& Chen, L. Fine tuning the immune response through B7-H3 and B7H4. Immunol. Rev. 229, 145-151 (2009).

23. Shen, Z. et al. Cloned dendritic cells can present exogenous antigens on both MHC class I and class II molecules. J. Immunol. 158, 2723-2730 (1997).

24. Qiao, B. Induction of systemic lupus erythematosus-like syndrome in syngeneic mice by immunization with activated lymphocyte-derived DNA. Rheumatology 44, 1108-1114 (2005).

25. Zhang, W., Xu, W. \& Xiong, S. Blockade of Notch1 Signaling Alleviates Murine Lupus via Blunting Macrophage Activation and M2b Polarization. J. Immunol. 184, 6465-6478 (2010).

26. Wen, Z. et al. Production of anti-double-stranded DNA antibodies in activated lymphocyte derived DNA induced lupus model was dependent on CD4+ T cells. Lupus 21, 508-516 (2012).

27. Watson, M. L. Genetic analysis of MRL-Ipr mice: relationship of the Fas apoptosis gene to disease manifestations and renal disease-modifying loci. J. Exp. Med. 176, 1645-1656 (1992).

28. Abroun, S. et al. STATs: an old story, yet mesmerizing. Cell J. 17, 395-411 (2015).

29. Wen, Z. K et al. DNA hypomethylation is crucial for apoptotic DNA to induce systemic lupus erythematosus-like autoimmune disease in SLE-nonsusceptible mice. Rheumatology 46, 1796-1803 (2007).

30. Zhang, W. et al. Amelioration of lupus nephritis by serum amyloid P component gene therapy with distinct mechanisms varied from different stage of the disease. PLOS ONE 6, e22659 (2011).

31. Lu, L. et al. Induction of anti-DNA antibodies by immunization with activated lymphocytes and active chromatin. Chin. Med. J. 111, 524-526 (1998).

32. Perry, D. et al. Murine models of systemic lupus erythematosus. J. Biomed Biotechnol. 2011, 271694 (2011).

33. Al-Janadi, M. et al. Cytokine profile in systemic lupus erythematosus, rheumatoid arthritis, and other rheumatic diseases. J. Clin. Immunol. 13, 58-67 (1993).

34. Tanaka, T. \& Kishimoto, T. Targeting interleukin-6: all the way to treat autoimmune and inflammatory diseases. Int. J. Biol. Sci. 8, 1227-1236 (2012).

35. Linker-Israeli, M. et al. Elevated levels of endogenous IL-6 in systemic lupus erythematosus. A putative role in pathogenesis. J. Immunol. 147, 117-123 (1991).

36. Hashiguchi, M. et al. Triggering receptor expressed on myeloid cell-like transcript 2 (TLT-2) is a counter-receptor for B7-H3 and enhances T cell responses. Proc. Natl Acad. Sci. USA 105, 10495-10500 (2008). 